Supporting Information

\title{
Monitoring DNA Structures by Dual Fluorescence of Pyrene Derivatives
}

Akimitsu Okamoto $^{* \dagger}$, Kazuki Tainaka, ${ }^{\dagger}$ Ken-ichiro Nishiza, ${ }^{\dagger}$ and Isao Saito ${ }^{* \dagger}$

'Department of Synthetic Chemistry and Biological Chemistry, Faculty of Engineering, Kyoto University,

Kyoto 615-8510, Japan.

${ }^{\ddagger}$ NEWCAT Institute, School of Engineering, Nihon University, and SORST, Japan Science and Technology

Agency, Tamura, Koriyama 963-8642, Japan

Phone: +81-75-383-2755

FAX: +81-75-753-2759

e-mail: okamoto@sbchem.kyoto-u.ac.jp 


\section{Materials and Methods}

General Methods and Materials. ${ }^{1} \mathrm{H}$ NMR spectra were measured with Varian Mercury 400 (400 MHz) spectrometer. ${ }^{13} \mathrm{C}$ NMR spectra were measured with JEOL JMN $\alpha-500(125 \mathrm{MHz})$ spectrometer. Coupling constant ( $J$ value) are reported in hertz. Chemical shifts are expressed in ppm downfield from tetramethylsilane, using residual chloroform $\left(\delta=7.24\right.$ in ${ }^{1} \mathrm{H} \mathrm{NMR}, \delta=77.0$ in $\left.{ }^{13} \mathrm{C} \mathrm{NMR}\right)$ as an internal standard. FAB mass spectra were recorded on JEOL JMS DX-300 spectrometer or JEOL JMS SX-102A spectrometer. Wakogel C-200 was used for silica gel column chromatography. Pre-coated TLC plates Merck silica gel $60 \mathrm{~F}_{254}$ was used for monitoring reactions. TLC spots were visualized with UV light or anisaldehyde (a solution of $9.0 \mathrm{~mL}$-anisaldehyde, $3.5 \mathrm{~mL}$ acetic acid and $10 \mathrm{~mL}$ sulfuric acid in $330 \mathrm{~mL}$ ethanol). All reagents and solvents were used as received. HPLC was performed on a CHEMCOBOND 5-ODS-H column $(10 \times 150 \mathrm{~mm})$ with a Gilson Chromatography Model 305 using UV detector Model 118 at $254 \mathrm{~nm}$. ESI mass spectra were recorded on a Perseptive Mariner ESI-TOF mass spectrometer. Masses of oligodeoxynucleotides were determined with a MALDI-TOF mass spectroscopy (acceleration voltage $21 \mathrm{kV}$, negative mode) with 2',3',4'-trihydroxyacetophenone as matrix, using $\mathrm{T}_{8}\left([\mathrm{M}-\mathrm{H}]^{-} 2370.61\right)$ and $\mathrm{T}_{17}\left([\mathrm{M}-\mathrm{H}]^{-}\right.$5108.37) as an internal standard. Shimadzu UV-2550 UV/vis spectrophotometer was used for absorption spectra measurement. Oligodeoxynucleotides were purchased from GeneDesign Inc., Japan. Calf intestinal alkaline phosphatase, Crotalus adamanteus Venom phosphodiesterase I, and Penicillium citrinum nuclease P1 were purchased from Promega, USB, and Roche, respectively. The reagents for the DNA synthesizer such as A, G, C, T- $\beta$-cyanoethyl phosphoramidites and CPG support were purchased from GLEN Research.

8-Acetyl-1-bromopyrene (S2). To a solution of 1-bromopyrene (S1) (6 g, $21 \mathrm{mmol})$ in dry dichloromethane $(200 \mathrm{~mL})$ was added aluminum chloride $(12 \mathrm{~g}, 90 \mathrm{mmol})$, and successively dropwise acetic anhydride $(15 \mathrm{~mL}$, $160 \mathrm{mmol}$ ) over $3.5 \mathrm{~h}$ under ice cooling. Upon stirring for an additional $23 \mathrm{~h}$, the reaction mixture was quenched by an addition of sat. $\mathrm{NaHCO}_{3}$. The aqueous mixture was extracted with chloroform three times, and then the combined organic phase was dried over $\mathrm{MgSO}_{4}$, filtered and evaporated. The crude product was purified by silica gel column chromatography (hexane : ethyl acetate $=10: 1$ ) to yield the mixture of $\mathbf{S 2}$ and 1,6-isomer (2.46 g, 36\%) as a yellow solid. S2 was further purified with LiChroprep ${ }^{\circledR}$ Si 60 (particle size 40-63 $\mu \mathrm{m}$, MERCK) using hexane-THF $(20: 1):{ }^{1} \mathrm{H}$ NMR $\left(\mathrm{CDCl}_{3}\right) \delta=9.02(\mathrm{~d}, 1 \mathrm{H}, J=9.7 \mathrm{~Hz}), 8.43(\mathrm{~d}, 1 \mathrm{H}$, $J=9.7 \mathrm{~Hz}), 8.31(\mathrm{~d}, 1 \mathrm{H}, J=8.1 \mathrm{~Hz}), 8.17(\mathrm{~d}, 1 \mathrm{H}, J=8.2 \mathrm{~Hz}), 8.07(\mathrm{~d}, 1 \mathrm{H}, J=8.1 \mathrm{~Hz}), 7.94(\mathrm{~d}, 2 \mathrm{H}, J=3.8$ $\mathrm{Hz}), 7.92(\mathrm{~d}, 1 \mathrm{H}, J=8.2 \mathrm{~Hz}), 2.87(\mathrm{~s}, 3 \mathrm{H}) ;{ }^{13} \mathrm{C} \mathrm{NMR}\left(\mathrm{CDCl}_{3}\right) \delta=201.8\left(\mathrm{C}_{\mathrm{C}=\mathrm{O}}\right), 133.8\left(\mathrm{C}_{5 \mathrm{a}}\right), 132.1\left(\mathrm{C}_{8}\right), 130.4$ $\left(\mathrm{C}_{2}\right), 130.3\left(\mathrm{C}_{3 \mathrm{a}}\right), 129.15\left(\mathrm{C}_{4}\right), 129.12\left(\mathrm{C}_{8 \mathrm{a}}\right), 128.9\left(\mathrm{C}_{10 \mathrm{a}}\right), 128.0\left(\mathrm{C}_{10}\right), 127.5\left(\mathrm{C}_{7}\right), 127.2\left(\mathrm{C}_{5}\right), 126.6\left(\mathrm{C}_{3}\right), 126.4$ $\left(\mathrm{C}_{9}\right), 125.2\left(\mathrm{C}_{10 \mathrm{~b}}\right), 124.6\left(\mathrm{C}_{6}\right), 124.2\left(\mathrm{C}_{10 \mathrm{c}}\right), 121.1\left(\mathrm{C}_{1}\right), 30.4\left(\mathrm{C}_{\mathrm{Me}}\right) ; \mathrm{MS}\left(\mathrm{FAB}, \mathrm{NBA} / \mathrm{CHCl}_{3}\right) \mathrm{m} / z(\%) 321[(\mathrm{M}+$ $\left.\mathrm{H})^{+}\right]$; HRMS (FAB) calcd. for $\mathrm{C}_{18} \mathrm{H}_{11} \mathrm{BrO}\left[(\mathrm{M}+\mathrm{H})^{+}\right]$321.9993, found 321.9992 .

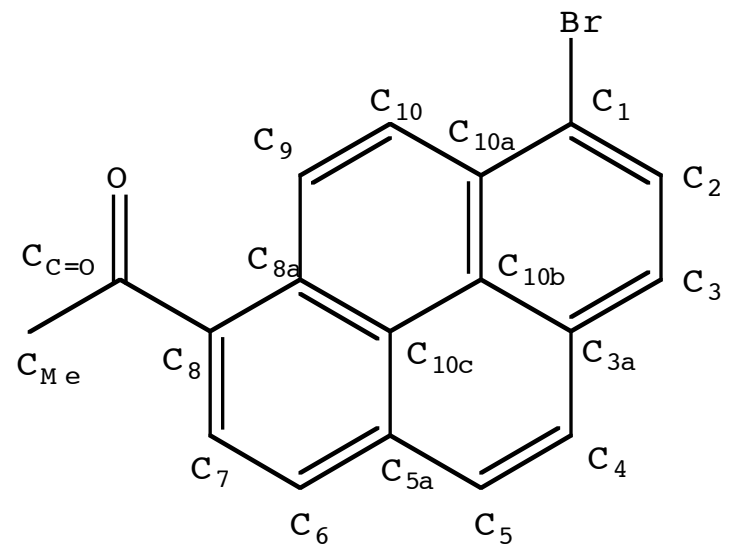

4-(4,4,5,5-Tetramethyl-1,3-dioxaborolan-2-yl)- $N, N$-dimethylaniline. To a solution of 4-bromo- $N, N$ dimethylaniline $(15 \mathrm{~g}, 75 \mathrm{mmol})$ and pinacolborane $(15.2 \mathrm{~mL}, 105 \mathrm{mmol})$ in dry $p$-dioxane $(200 \mathrm{~mL})$ was 
added $\mathrm{PdCl}_{2}\left(\mathrm{PPh}_{3}\right)_{2}(4 \mathrm{~g}, 5.7 \mathrm{mmol})$ and triethylamine $(31 \mathrm{~mL}, 222 \mathrm{mmol})$. The mixture was refluxed for $17 \mathrm{~h}$. The reaction mixture was evaporated, and diluted with brine. The aqueous mixture was extracted with chloroform three times, and then the combined organic phase was dried over $\mathrm{MgSO}_{4}$, filtered and evaporated. The crude product was purified by silica gel column chromatography (hexane : ethyl acetate $=70: 1$ ) to yield 4-(4,4,5,5-tetramethyl-1,3-dioxaborolan-2-yl)- $N, N$-dimethylaniline $(13.1 \mathrm{~g}, 71 \%)$ as a white solid: ${ }^{1} \mathrm{H}$ NMR $\left(\mathrm{CDCl}_{3}\right) \delta=7.70(\mathrm{~d}, 2 \mathrm{H}, J=9.2 \mathrm{~Hz}), 6.70(\mathrm{~d}, 2 \mathrm{H}, J=8.8 \mathrm{~Hz}), 2.99(\mathrm{~s}, 6 \mathrm{H}), 1.33(\mathrm{~s}, 12 \mathrm{H}) ;{ }^{13} \mathrm{C} \mathrm{NMR}\left(\mathrm{CDCl}_{3}\right)$ $\delta=152.5,136.1,111.3,83.1,40.1,24.8 ; \mathrm{MS}\left(\mathrm{FAB}, \mathrm{NBA} / \mathrm{CHCl}_{3}\right) m / z(\%) 247\left[(\mathrm{M}+\mathrm{H})^{+}\right]$; HRMS (FAB) calcd. for $\mathrm{C}_{14} \mathrm{H}_{22} \mathrm{BNO}_{2}\left[(\mathrm{M}+\mathrm{H})^{+}\right] 247.1738$, found 247.1744 .

1-Acetyl-8-(4- $\boldsymbol{N}, \boldsymbol{N}$-dimethylaminophenyl)pyrene (S3). To a mixture of $\mathbf{S 2}$ (516 mg, $1.6 \mathrm{mmol})$, 4-(4,4,5,5tetramethyl-1,3-dioxaborolan-2-yl)- $N, N$-dimethylaniline $(435 \mathrm{mg}, 1.76 \mathrm{mmol})$, toluene $(10 \mathrm{~mL})$, and aqueous sodium hydroxide solution $(1 \mathrm{M}, 5 \mathrm{~mL})$, tetrakis(triphenylphosphine)palladium (370 $\mathrm{mg}, 0.32 \mathrm{mmol})$ was added, and the mixture was viscously stirred under reflux for $20 \mathrm{~h}$. The layers were then separated, the aqueous phase was extracted with toluene three times, the combined organic phase was dried with $\mathrm{MgSO}_{4}$, and the solvent was evaporated. The residue was purified by column chromatography on silica gel (hexane : toluene $=1: 20)$ to give $356 \mathrm{mg}(69 \%)$ of $\mathbf{S 3}$ as an orange solid: ${ }^{1} \mathrm{H} \mathrm{NMR}\left(\mathrm{CDCl}_{3}\right) \delta=8.97(\mathrm{~d}, 1 \mathrm{H}, J=9.9$ $\mathrm{Hz}), 8.43(\mathrm{~d}, 1 \mathrm{H}, J=9.9 \mathrm{~Hz}), 8.38(\mathrm{~d}, 1 \mathrm{H}, J=8.1 \mathrm{~Hz}), 8.25(\mathrm{~d}, 1 \mathrm{H}, J=7.9 \mathrm{~Hz}), 8.17(\mathrm{~d}, 1 \mathrm{H}, J=8.6 \mathrm{~Hz}), 8.15$ $(\mathrm{d}, 1 \mathrm{H}, J=6.0 \mathrm{~Hz}), 8.05(\mathrm{~d}, 1 \mathrm{H}, J=7.7 \mathrm{~Hz}), 8.03(\mathrm{~d}, 1 \mathrm{H}, J=7.5 \mathrm{~Hz}), 7.54(\mathrm{~d}, 1 \mathrm{H}, J=8.2 \mathrm{~Hz}), 6.92(\mathrm{~d}, 1 \mathrm{H}, J$ $=6.8 \mathrm{~Hz}), 3.07(\mathrm{~s}, 6 \mathrm{H}), 2.90(\mathrm{~s}, 3 \mathrm{H}) ;{ }^{13} \mathrm{C} \mathrm{NMR}\left(\mathrm{CDCl}_{3}\right) \delta=202.1,139.5,136.1,134.5,131.5,131.4,129.84$, 129.78, 129.6, 128.3, 128.2, 127.9, 127.2, 126.6, 126.1, 125.8, 125.5, 124.8, 124.6, 123.8, 112.4, 40.6, 30.4; MS (FAB, NBA / $\left.\mathrm{CDCl}_{3}\right) \mathrm{m} / z(\%) 363\left[(\mathrm{M}+\mathrm{H})^{+}\right]$; HRMS (FAB / $\mathrm{CDCl}_{3}$ ) calcd. for $\mathrm{C}_{26} \mathrm{H}_{21} \mathrm{NO}\left[(\mathrm{M}+\mathrm{H})^{+}\right]$ 363.1623 , found 363.1626 .

8-(4- $\boldsymbol{N}, \boldsymbol{N}$-Dimethylaminophenyl)pyrene-1-carboxylic acid (S4). Sodium hydroxide (0.24 g, 6 mmol) was dissolved in $6 \mathrm{~mL}$ of water. The solution was cooled to $0{ }^{\circ} \mathrm{C}$, and bromine $(84 \mu \mathrm{L}, 1.6 \mathrm{mmol})$ was added. The yellow solution was diluted with $6 \mathrm{~mL}$ of 1,4-dioxane. After the solution was stirred at $0{ }^{\circ} \mathrm{C}$ for $10 \mathrm{~min}$, a precooled solution of $\mathbf{S 3}(150 \mathrm{mg}, 0.41 \mathrm{mmol})$ dissolved in $6 \mathrm{~mL}$ of 1,4-dioxane was added. The solution was stirred at $0{ }^{\circ} \mathrm{C}$ for $3 \mathrm{~h}$. Saturated $\mathrm{Na}_{2} \mathrm{SO}_{3}(2 \mathrm{~mL})$ was added, and stirring was continued at room temperature for $1 \mathrm{~h}$. After acidification with $10 \%$ hydrochloric acid, the reaction mixture was transferred into a separatory funnel, and extracted with chloroform three times. The combined organic phase was dried with $\mathrm{MgSO}_{4}$, and the solvent was evaporated. An orange solid S4 was isolated (192 $\mathrm{mg}, 93 \%)$. The product was used directly in the next step with no further purification.

$\boldsymbol{N}$-Propargyl 8-(4- $\boldsymbol{N}^{\prime}, \boldsymbol{N}^{\prime}$-dimethylaminophenyl)pyrene-1-carboxamide (1). A solution of $\mathbf{S 4}$ (130 mg, 0.36 $\mathrm{mmol}$ ) and PyBOP (260 mg, $0.50 \mathrm{mmol}$ ) in anhydrous DMF (3 mL) was stirred at room temperature. After 15 min, propargylamine $(50 \mu \mathrm{L}, 0.73 \mathrm{mmol}$ ) was added, and the mixture was stirred at room temperature for 2.5 h. The resulting mixture was concentrated in vacuo, and then diluted with ethyl acetate. This solution was washed with sat. $\mathrm{NH}_{4} \mathrm{Cl}$, brine, and water. The combined organic phase was dried over $\mathrm{MgSO}_{4}$, filtered and evaporated. The residue was purified by column chromatography on silica gel (hexane : chloroform $=1: 1$ ) to give $120 \mathrm{mg}(83 \%)$ of 1 as a yellow solid: ${ }^{1} \mathrm{H} \mathrm{NMR}\left(\mathrm{CDCl}_{3}\right) \delta=8.49(\mathrm{~d}, 1 \mathrm{H}, J=9.7 \mathrm{~Hz}), 8.36(\mathrm{~d}, 1 \mathrm{H}, J=9.7$ $\mathrm{Hz}), 8.22(\mathrm{~d}, 1 \mathrm{H}, J=7.9 \mathrm{~Hz}), 8.11(\mathrm{~d}, 1 \mathrm{H}, J=9.0 \mathrm{~Hz}), 8.09(\mathrm{~d}, 2 \mathrm{H}, J=3.9 \mathrm{~Hz}), 8.02(\mathrm{~d}, 1 \mathrm{H}, J=1.8 \mathrm{~Hz}), 8.00$ $(\mathrm{d}, 1 \mathrm{H}, J=5.9 \mathrm{~Hz}), 7.51(\mathrm{~d}, 2 \mathrm{H}, J=8.8 \mathrm{~Hz}), 6.92(\mathrm{~d}, 2 \mathrm{H}, J=8.6 \mathrm{~Hz}), 6.29(\mathrm{t}(\mathrm{br}), 1 \mathrm{H}), 4.41(\mathrm{dd}, 2 \mathrm{H}, J=2.6$, $2.7 \mathrm{~Hz}), 3.06(\mathrm{~s}, 6 \mathrm{H}), 2.31(\mathrm{t}, 1 \mathrm{H}, J=2.4 \mathrm{~Hz}) ;{ }^{13} \mathrm{C} \mathrm{NMR}\left(\mathrm{CDCl}_{3}\right) \delta=169.5,149.9,139.2,133.2,131.4,129.9$, 129.4, 128.9, 128.7, 128.2, 128.0, 127.4, 126.6, 125.7, 125.2, 124.9, 124.7, 124.1, 123.8, 120.2, 112.3, 79.5, 72.0, 40.6, 30.0; MS (FAB, NBA / $\left.\mathrm{CHCl}_{3}\right) \mathrm{m} / z(\%) 402\left[(\mathrm{M}+\mathrm{H})^{+}\right]$; HRMS (FAB) calcd. for $\mathrm{C}_{28} \mathrm{H}_{22} \mathrm{~N}_{2} \mathrm{O}\left[\left(\mathrm{M}^{2}\right.\right.$ $\left.+\mathrm{H})^{+}\right]$402.1732, found 402.1735 .

5-[3-(8-(4- $N^{\prime}, N^{\prime}$-Dimethylaminophenyl)pyrene-1-carboxamido)-1-propynyl]-3',5'-O,O'-bis(tertbutyldimethylsilyl)-2'-deoxyuridine (S5). To a solution of 5-iodo-3',5'-O,O'-bis(tert-butyldimethylsilyl)-2'deoxyuridine (146 mg, $0.25 \mathrm{mmol}), 1$ (90 mg, $0.25 \mathrm{mmol})$, and triethylamine $(115 \mu \mathrm{L}, 0.82 \mathrm{mmol})$ in $2 \mathrm{~mL} \mathrm{of}$ anhydrous DMF were added tetrakis(triphenylphosphine)palladium (57 mg, $0.049 \mathrm{mmol}$ ) and copper(I) iodide (20 mg, $0.11 \mathrm{mmol}$ ). The mixture was stirred at room temperature for $12 \mathrm{~h}$. The resulting mixture was concentrated in vacuo, and diluted with ethyl acetate. The solution was washed with 5\% EDTA solution twice 
and then $5 \%$ sodium bisulfite solution, dried over $\mathrm{Na}_{2} \mathrm{SO}_{4}$, filtered and evaporated. The crude product was purified by silica gel column chromatography (chloroform : methanol = $90: 1)$ to yield $\mathbf{S 5}(121 \mathrm{mg}, 56 \%)$ as a yellow solid: ${ }^{1} \mathrm{H}$ NMR $\left(\mathrm{CDCl}_{3}\right) \delta=8.54(\mathrm{~d}, 1 \mathrm{H}, J=9.6 \mathrm{~Hz}), 8.23(\mathrm{~d}, 1 \mathrm{H}, J=7.6 \mathrm{~Hz}), 8.13(\mathrm{~d}, 1 \mathrm{H}, J=4.8$ $\mathrm{Hz}), 8.117(\mathrm{~d}, 1 \mathrm{H}, J=4.0 \mathrm{~Hz}), 8.116(\mathrm{~s}, 1 \mathrm{H}), 8.05(\mathrm{~d}, 2 \mathrm{H}, J=1.6 \mathrm{~Hz}), 8.02(\mathrm{~d}, 1 \mathrm{H}, J=5.2 \mathrm{~Hz}), 8.01(\mathrm{~d}, 1 \mathrm{H}, J$ $=6.0 \mathrm{~Hz}), 7.66(\mathrm{~d}, 2 \mathrm{H}, J=12.0 \mathrm{~Hz}), 6.98(\mathrm{~s},(\mathrm{br}), 2 \mathrm{H}), 6.44(\mathrm{~s}(\mathrm{br}), 1 \mathrm{H}), 6.26(\mathrm{dd}, 1 \mathrm{H}, J=1.6,6.0 \mathrm{~Hz}), 4.62$ $(\mathrm{d}, 2 \mathrm{H}, J=5.2 \mathrm{~Hz}), 4.40(\mathrm{dt}, 1 \mathrm{H}, J=2.4,6.0 \mathrm{~Hz}), 3.96(\mathrm{~d}, 1 \mathrm{H}, J=2.4 \mathrm{~Hz}), 3.88(\mathrm{dd}, 1 \mathrm{H}, J=2.4,11.6 \mathrm{~Hz})$, $3.75(\mathrm{dd}, 1 \mathrm{H}, J=2.0,9.2 \mathrm{~Hz}), 2.94(\mathrm{~s}, 3 \mathrm{H}), 2.88(\mathrm{~s}, 3 \mathrm{H}), 2.31$ (ddd, 1H, $J=2.8,3.2,13.2 \mathrm{~Hz}), 1.98-2.05(\mathrm{~m}$, $1 \mathrm{H}), 0.89(\mathrm{~s}, 9 \mathrm{H}), 0.88(\mathrm{~s}, 9 \mathrm{H}), 0.13(\mathrm{~s}, 3 \mathrm{H}), 0.11(\mathrm{~s}, 3 \mathrm{H}), 0.075(\mathrm{~s}, 3 \mathrm{H}), 0.068(\mathrm{~s}, 3 \mathrm{H}) ;{ }^{13} \mathrm{C} \mathrm{NMR}\left(\mathrm{CDCl}_{3}\right) \delta=$ $169.3,166.4,162.5,161.2,156.2,148.9,143.2,133.2,132.2,132.1,131.89,131.86,131.6,128.9,128.5$, $128.4,128.1,126.8,125.7,125.2,124.8,88.5,85.9,75.2,72.4,63.0,44.3,42.1,36.4,30.9,25.97,25.72,18.4$, 18.0, 7.4, -4.7, -4.9, -5.3, -5.5; MS (FAB, NBA / $\left.\mathrm{CHCl}_{3}\right) m / z(\%) 857\left[(\mathrm{M}+\mathrm{H})^{+}\right]$; HRMS (FAB) calcd. for $\mathrm{C}_{49} \mathrm{H}_{60} \mathrm{~N}_{4} \mathrm{O}_{6} \mathrm{Si}_{2}\left[(\mathrm{M}+\mathrm{H})^{+}\right]$856.4051, found 856.4054.

5-[3-(8-(4- $N^{\prime}, N^{\prime}$-Dimethylaminophenyl)pyrene-1-carboxamido)-1-propynyl]-2'-deoxyuridine (2). To a solution of $\mathbf{S 5}(20 \mathrm{mg}, 0.023 \mathrm{mmol})$ in tetrahydrofuran $(1.2 \mathrm{~mL})$ was added $1.0 \mathrm{M}$ solution of TBAF in tetrahydrofuran $(300 \mu \mathrm{L}, 0.3 \mathrm{mmol})$. The mixture was stirred at room temperature for $2 \mathrm{~h}$. The mixture was concentrated in vacuo. The crude product was purified by silica gel column chromatography (chloroform : methanol $=40: 1)$ to yield $2(14 \mathrm{mg}, 95 \%)$ as a white solid: ${ }^{1} \mathrm{H}$ NMR $\left(\mathrm{CDCl}_{3}\right) \delta=8.54(\mathrm{~s}, 1 \mathrm{H}), 8.49(\mathrm{~d}, 1 \mathrm{H}, J$ $=10.0 \mathrm{~Hz}), 8.26(\mathrm{~d}, 1 \mathrm{H}, J=9.6 \mathrm{~Hz}), 8.19(\mathrm{~d}, 1 \mathrm{H}, J=8.0 \mathrm{~Hz}), 8.12(\mathrm{~d}, 1 \mathrm{H}, J=7.6 \mathrm{~Hz}), 8.03(\mathrm{~d}, 1 \mathrm{H}, J=8.0$ $\mathrm{Hz}), 7.99(\mathrm{~d}, 1 \mathrm{H}, J=9.2 \mathrm{~Hz}), 7.93(\mathrm{~d}, 1 \mathrm{H}, J=8.0 \mathrm{~Hz}), 7.89(\mathrm{~d}, 1 \mathrm{H}, J=9.2 \mathrm{~Hz}), 7.44(\mathrm{~d}, 2 \mathrm{H}, J=8.8 \mathrm{~Hz}), 6.85$ $(\mathrm{d}, 2 \mathrm{H}, J=8.8 \mathrm{~Hz}), 6.05(\mathrm{dd}, 1 \mathrm{H}, J=4.0,5.6 \mathrm{~Hz}), 4.58(\mathrm{~d}, 1 \mathrm{H}, J=5.6 \mathrm{~Hz}), 4.52(\mathrm{~d}, 2 \mathrm{H}, J=4.4 \mathrm{~Hz}), 3.91(\mathrm{~d}$, $1 \mathrm{H}, J=10.8 \mathrm{~Hz}), 3.81(\mathrm{t}, 3 \mathrm{H}, J=4.8 \mathrm{~Hz}), 3.01(\mathrm{~s}, 6 \mathrm{H}), 2.35$ (quin, $1 \mathrm{H}, J=7.2 \mathrm{~Hz}), 2.11-2.17(\mathrm{~m}, 1 \mathrm{H}) ;{ }^{13} \mathrm{C}$ NMR $\left(\mathrm{CDCl}_{3}\right) \delta=169.3,161.9,149.8,149.2,145.0,138.9,132.8,131.3,129.7,129.5,128.7,128.6,128.4$, $128.0,127.8,126.8,126.5,125.47,125.43,124.9,124.8,124.3,124.2,112.3,98.6,89.1,87.7,85.4,77.2$, 75.1, 68.3, 60.2, 44.2, 40.4, 30.7; MS (FAB, DTT, TG/CDCl 3 ) m/z (\%) $629\left[(\mathrm{M}+\mathrm{H})^{+}\right]$; HRMS (FAB) calcd. for $\mathrm{C}_{37} \mathrm{H}_{32} \mathrm{~N}_{4} \mathrm{O}_{6}\left[(\mathrm{M}+\mathrm{H})^{+}\right]$628.2322, found 628.2339.

\section{5-[3-(8-(4- $N^{\prime}, N^{\prime}$-Dimethylaminophenyl)pyrene-1-carboxamido)-1-propynyl]-5'-O-(4,4'-}

dimethoxytrityl)-2'-deoxyuridine (S6). To a solution of 5-iodo-5'-O-(4,4'-dimethoxytrityl)-2'-deoxyuridine (158 mg, $0.24 \mathrm{mmol}), 1$ (92.6 mg, $0.23 \mathrm{mmol})$, and triethylamine (92 $\mu \mathrm{L}, 0.66 \mathrm{mmol})$ in $3 \mathrm{~mL}$ of anhydrous DMF were added tetrakis(triphenylphosphine)palladium (46 mg, $0.04 \mathrm{mmol}$ ) and copper(I) iodide (15 mg, $0.079 \mathrm{mmol})$. The mixture was stirred at room temperature for $17 \mathrm{~h}$. The resulting mixture was concentrated in vacuo, and diluted with ethyl acetate. The solution was washed with 5\% EDTA solution twice and then 5\% sodium bisulfite solution, dried over $\mathrm{Na}_{2} \mathrm{SO}_{4}$, filtered, and evaporated. The crude product was purified by silica gel column chromatography (chloroform : methanol $=50: 1)$ to yield $\mathbf{S 6}(152 \mathrm{mg}, 71 \%)$ as a brown solid: ${ }^{1} \mathrm{H}$ NMR $\left(\mathrm{CDCl}_{3}\right) \delta=8.73(\mathrm{~d}, 1 \mathrm{H}, J=8.4 \mathrm{~Hz}), 8.43(\mathrm{~d}, 1 \mathrm{H}, J=8.0 \mathrm{~Hz}), 8.30(\mathrm{~d}, 1 \mathrm{H}, J=10.0 \mathrm{~Hz}), 8.18$ $(\mathrm{d}, 1 \mathrm{H} J=8.0 \mathrm{~Hz}), 8.10(\mathrm{~s}, 1 \mathrm{H}), 8.06(\mathrm{~d}, 1 \mathrm{H}, J=8.8 \mathrm{~Hz}), 8.04(\mathrm{~d}, 1 \mathrm{H}, J=3.2 \mathrm{~Hz}), 7.99(\mathrm{~d}, 1 \mathrm{H}, J=8.0 \mathrm{~Hz})$, $7.94(\mathrm{~d}, 1 \mathrm{H}, J=9.2 \mathrm{~Hz}), 7.40(\mathrm{~d}, 2 \mathrm{H}, J=6.8 \mathrm{~Hz}), 7.21-7.35(\mathrm{~m}, 13 \mathrm{H}), 6.84(\mathrm{~d}, 2 \mathrm{H}, J=9.2 \mathrm{~Hz}), 6.21(\mathrm{dd}, 1 \mathrm{H}$, $J=2.4,4.0 \mathrm{~Hz}), 5.70(\mathrm{~s}, 1 \mathrm{H}), 4.65(\mathrm{t}, 1 \mathrm{H}, J=5.2 \mathrm{~Hz}), 4.54(\mathrm{~d}, 2 \mathrm{H}, J=2.8 \mathrm{~Hz}), 4.11(\mathrm{dt}, 1 \mathrm{H}, J=2.8,5.2 \mathrm{~Hz})$, $3.69(\mathrm{~s}, 3 \mathrm{H}), 3.68(\mathrm{~s}, 3 \mathrm{H}), 3.45(\mathrm{dd}, 1 \mathrm{H}, J=2.8,3.2 \mathrm{~Hz}), 3.36(\mathrm{dd}, 1 \mathrm{H}, J=3.2,3.6 \mathrm{~Hz}), 3.05(\mathrm{~s}, 6 \mathrm{H}), 2.47$ $(\mathrm{ddd}, 1 \mathrm{H}, J=2.8,3.2,13.6 \mathrm{~Hz}), 2.21-2.35(\mathrm{~m}, 1 \mathrm{H})$; MS $\left(\mathrm{FAB}, \mathrm{NBA} / \mathrm{CHCl}_{3}\right) \mathrm{m} / z(\%) 930\left[(\mathrm{M}+\mathrm{H})^{+}\right]$ HRMS (FAB) calcd. for $\mathrm{C}_{58} \mathrm{H}_{50} \mathrm{~N}_{4} \mathrm{O}_{8}\left[(\mathrm{M}+\mathrm{H})^{+}\right]$930.3629, found 930.3615.

\section{5-[3-(8-(4- $N^{\prime}, N^{\prime}$-Dimethylaminophenyl)pyrene-1-carboxamido)-1-propynyl]-5'-O-(4,4'-}

dimethoxytrityl)-2'-deoxyuridine 3'-O-(2-cyanoethyl $\boldsymbol{N}^{\prime \prime}, \boldsymbol{N}^{\prime \prime}$-diisopropylphosphoramidite) (S7). To a solution of S6 $(76 \mathrm{mg}, 0.08 \mathrm{mmol})$ and tetrazole $(6 \mathrm{mg}, 0.086 \mathrm{mmol})$ in anhydrous acetonitrile $(1.0 \mathrm{~mL})$ was added 2-cyanoethyl tetraisopropylphosphorodiamidite $(30 \mu \mathrm{L}, 0.094 \mathrm{mmol})$. The mixture was stirred at room temperature for $30 \mathrm{~min}$. The mixture was filtered and used with no further purification for DNA synthesis.

Oligonucleotide synthesis and characterization. Oligodeoxynucleotide sequences were synthesized by conventional phosphoramidite method by using an Applied Biosystems 392 DNA/RNA synthesizer. Oligodeoxynucleotides were purified by reverse phase HPLC on a 5-ODS-H column $(10 \times 150 \mathrm{~mm})(\mathrm{elution}$ with a solvent mixture of $0.1 \mathrm{M}$ triethylamine acetate (TEAA) buffer, $\mathrm{pH} 7.0$, linear gradient $0-50 \%$ 
acetonitrile $(0-50 \mathrm{~min})$ at a flow rate $3.0 \mathrm{~mL} / \mathrm{min})$. Oligodeoxynucleotides containing 2 were fully digested with calf intestine alkaline phosphatase $(50 \mathrm{U} / \mathrm{mL})$, snake venom phosphodiesterase $(0.15 \mathrm{U} / \mathrm{mL})$ and $\mathrm{P} 1$ nuclease $(50 \mathrm{U} / \mathrm{mL})$ at $37^{\circ} \mathrm{C}$ for $10 \mathrm{~h}$. Digested solutions were analyzed by HPLC on a 5-ODS-H column (4.6 $\times 150 \mathrm{~mm}$ ) (elution with a solvent mixture of $0.1 \mathrm{M}$ triethylammonium acetate buffer, $\mathrm{pH} 7.0$, linear gradient $5-13 \%$ acetonitrile $(0-8 \mathrm{~min}), 13-100 \%$ acetonitrile $(8-20 \mathrm{~min})$ at a flow rate $1.0 \mathrm{~mL} / \mathrm{min})$. Concentration of each oligodeoxynucleotide was determined by comparing peak areas with standard solution containing dA, $\mathrm{dC}, \mathrm{dG}$, and $\mathrm{dT}$ at a concentration of $0.1 \mathrm{mM}$. The isolated yield of $\mathbf{3}$ was $33 \%$.

Fluorescence Measurement. All fluorescence spectra were taken in a buffer containing $0.1 \mathrm{M}$ sodium chloride and $50 \mathrm{mM}$ phosphate, $\mathrm{pH}$ 7.0. Fluorescence spectra were obtained using a SHIMADZU RF-5300PC.

UV Measurement. All UV spectra were taken in a buffer containing $0.1 \mathrm{M}$ sodium chloride and $50 \mathrm{mM}$ phosphate, pH 7.0 using a Shimadzu UV-2550 UV/vis spectrophotometer.

Stevens-Ban plot. Absorption and fluorescence spectra of $7.3 \quad \mu \mathrm{M} \quad 2,25 \quad \mu \mathrm{M}$ single strand oligodeoxynucleotide $\mathbf{3}$, and $25 \mu \mathrm{M}$ duplex $\mathbf{3} / \mathbf{3}^{\prime}$ were measured in $50 \mathrm{mM}$ sodium phosphate $(\mathrm{pH}=7.0)$ and $0.1 \mathrm{M}$ sodium chloride. The data were plotted for each $5{ }^{\circ} \mathrm{C}$ rise from $5{ }^{\circ} \mathrm{C}$ to $75{ }^{\circ} \mathrm{C}$. In a plot of $\ln \left(\Phi^{\prime}(\mathrm{ICT}) / \Phi(\mathrm{LE})\right)$ versus the reciprocal absolute temperature, a linear dependence is obtained at the highand low-temperature limits, for which the conditions $k_{\mathrm{d}}>1 / \tau_{0}$ (HTL) and $k_{\mathrm{d}}<<1 / \tau_{0}$ (LTL) hold. The slopes of these straight lines are $\left(E_{\mathrm{d}}-E_{\mathrm{a}}\right) / R=-\Delta H / R$ (for HTL) and $-E_{\mathrm{a}} / R$ (for LTL), when $k_{\mathrm{f}}^{\prime} / k_{\mathrm{f}}$ and $\tau_{0}$ are independent of temperature. The general expressions, however, for these slopes are $-\Delta H / R+\mathrm{d} \ln \left(k_{\mathrm{f}}^{\prime} / k_{\mathrm{f}}\right) / \mathrm{d}(1 / \mathrm{T})$ under HTL conditions and $-E_{\mathrm{a}} / R+\mathrm{d} \ln \left(k_{\mathrm{f}}^{\prime} / k_{\mathrm{f}}\right) / \mathrm{d}(1 / \mathrm{T})-\mathrm{d} \ln \left(1 / \tau_{0}\right) / \mathrm{d}(1 / \mathrm{T})$ for LTL conditions. Here, $E_{\mathrm{a}}$ and $E_{\mathrm{d}}$ are the activation energies of $k_{\mathrm{a}}$ and $k_{\mathrm{d}}$, respectively, whereas $\Delta H$ is the enthalpy change associated with the ICT formation. 


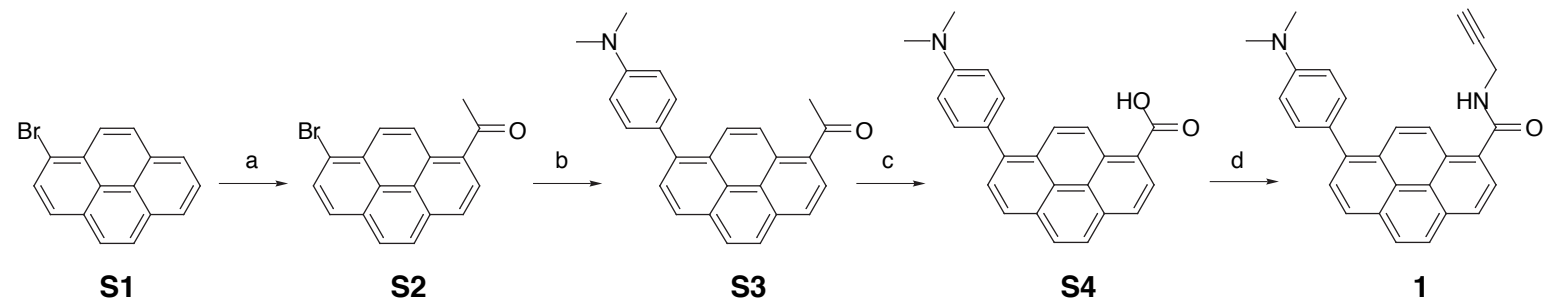

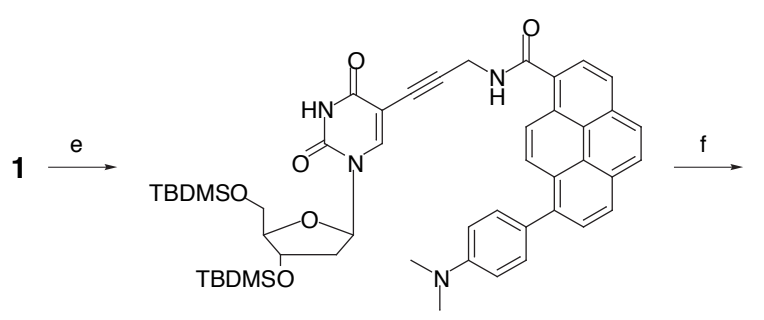

S5

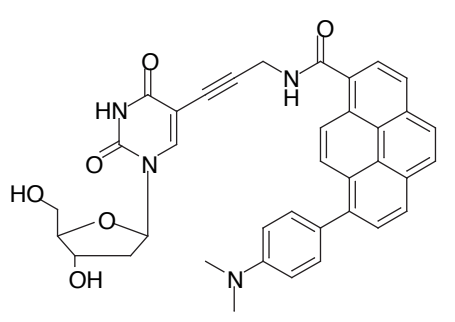

2

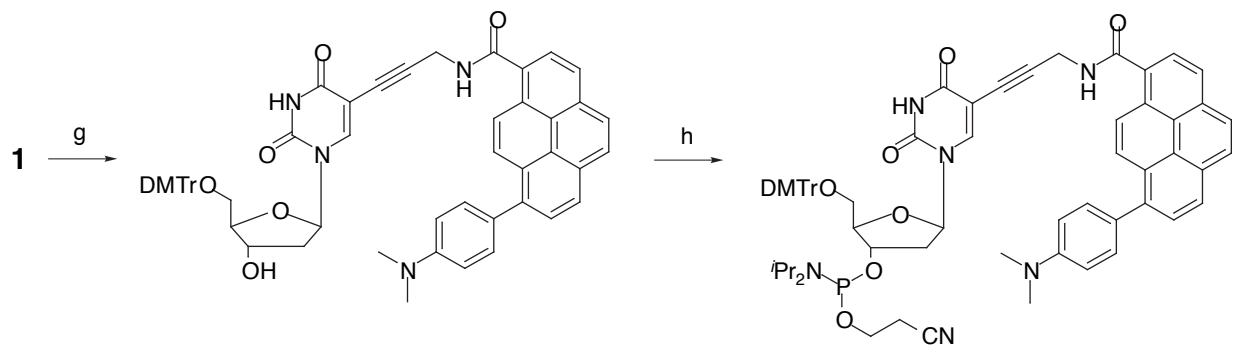

S6

S7

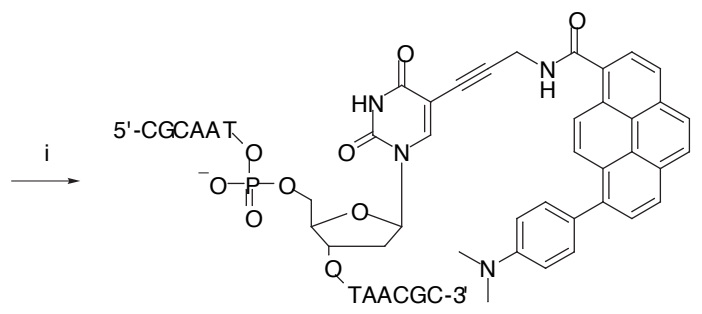

3

Scheme S1. Synthesis of the oligodeoxynucleotide containing a fluorophore with dual fluorescence. (a) aluminium chloride, acetic anhydride, dichloromethane, $0{ }^{\circ} \mathrm{C}, 26 \mathrm{~h}, 36 \%$; (b) 4-(4,4,5,5-tetramethyl-1,3dioxaborolan-2-yl)- $N, N$-dimethylaniline, $\mathrm{Pd}\left(\mathrm{PPh}_{3}\right)_{4}$, sodium hydroxide, toluene, reflux, $20 \mathrm{~h}, 69 \%$; (c) bromine, sodium hydroxide, water, dioxane, $0{ }^{\circ} \mathrm{C}, 3 \mathrm{~h}, 93 \%$; (d) propargylamine, PyBOP, DMF, room temperature, $2.5 \mathrm{~h}, 83 \%$; (e) 5-iodo-3',5'-O,O'-bis(tert-butyldimethylsilyl)-2'-deoxyuridine, $\mathrm{Pd}\left(\mathrm{PPh}_{3}\right)_{4}, \mathrm{CuI}$, triethylamine, DMF, room temperature, $12 \mathrm{~h}, 56 \%$; (f) tetrabutylammonium fluoride, THF, room temperature, 2 h, 95\%; (g) 5-iodo-5'-O-(4,4'-dimethoxytrityl)-2'-deoxyuridine, $\mathrm{Pd}\left(\mathrm{PPh}_{3}\right)_{4}, \mathrm{CuI}$, triethylamine, DMF, room temperature, $17 \mathrm{~h}, 71 \%$; (h) 2-cyanoethyl tetraisopropylphosphorodiamidite, tetrazole, acetonitrile, room temperature, 30 min, quant.; (i) DNA synthesizer, and then $28 \%$ ammonia, $55^{\circ} \mathrm{C}, 8 \mathrm{~h}$. 


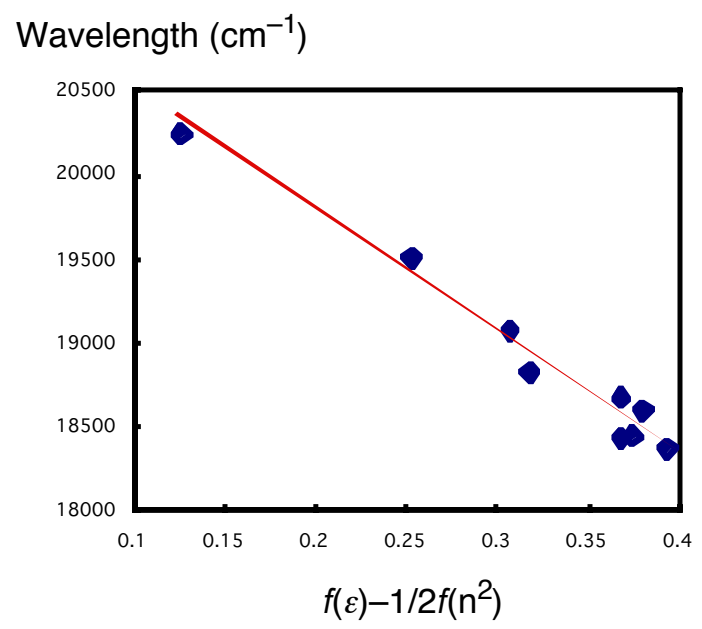

Figure S1. An approximately linear relationship between the fluorescence maxima of $\mathbf{1}$ and solvent polarity parameters. The polarity parameter in the ICT state is follows:

$$
\begin{aligned}
& v_{\max }(\mathrm{flu})=-2 / h c \rho^{3} \bullet \mu_{\mathrm{e}}\left(\mu_{\mathrm{e}}-\mu_{\mathrm{g}} \bullet \cdot\left(f(\varepsilon)-1 / 2 \bullet f\left(n^{2}\right)\right)+\mathrm{const}\right. \\
& f(\varepsilon)-1 / 2 \bullet f\left(n^{2}\right)=(\varepsilon-1) /(2 \varepsilon+1)-\left(n^{2}-1\right) / 2\left(2 n^{2}+1\right)
\end{aligned}
$$

The straight line in the figure suggests that the fluorescence originates from ICT state. The ground-state dipole moment $\mu_{\mathrm{g}}$ and Onsager radius $\rho$ of $\mathbf{1}$ at the ground state are $4.76 \mathrm{D}$ and $7.0 \AA$, respectively. Therefore, we obtained the dipole moment at the excited state, 18.1 D. 
(a)

Abs

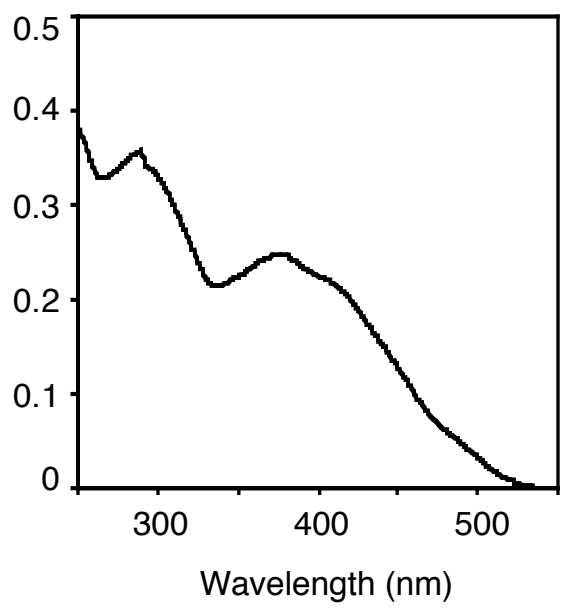

(b)

Abs

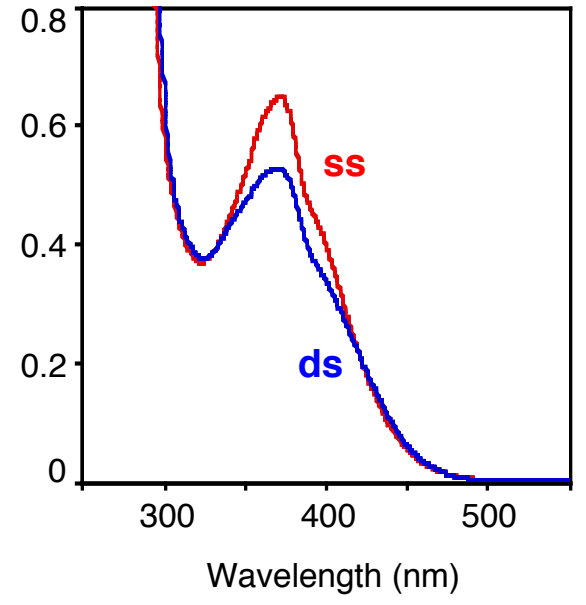

Figure S2. (a) Absorption spectrum of $7.3 \mu \mathrm{M} 2$ in $50 \mathrm{mM}$ sodium phosphate $(\mathrm{pH}=7.0)$ and $0.1 \mathrm{M}$ sodium chloride at $25^{\circ} \mathrm{C}$. (b) Absorption spectra of $25 \mu \mathrm{M}$ single strand oligodeoxynucleotide 3 (red line), and 25 $\mu \mathrm{M}$ duplex $\mathbf{3} / \mathbf{3}^{\prime}$ (blue line) in $50 \mathrm{mM}$ sodium phosphate $(\mathrm{pH}=7.0)$ and $0.1 \mathrm{M}$ sodium chloride at $25^{\circ} \mathrm{C}$. 


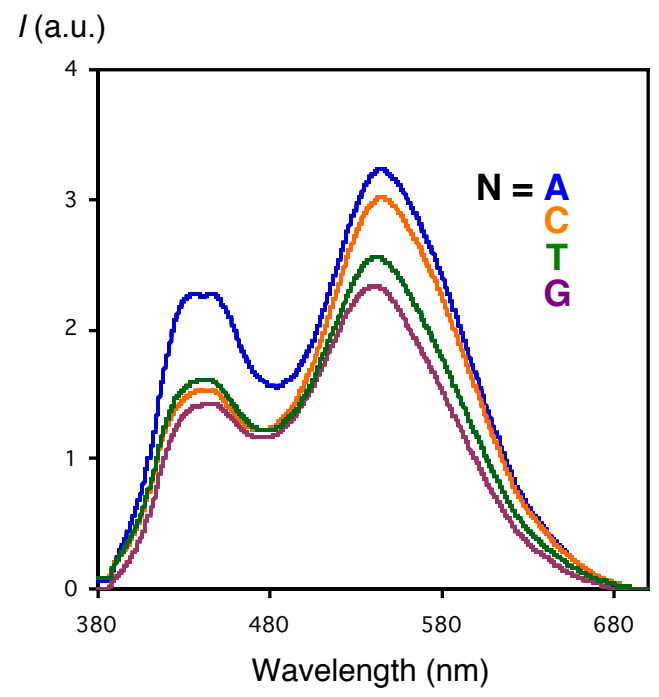

Figure S3. Fluorescence spectra of $25 \mu \mathrm{M}$ duplexes, 5'-d(CGCAAT2TAACGC)-3'/5'-d(GCGTTANATTG$\mathrm{CG})-3^{\prime}(\mathrm{N}=\mathrm{A}, \mathrm{G}, \mathrm{C}$, or $\mathrm{T})$, in $50 \mathrm{mM}$ sodium phosphate $(\mathrm{pH}=7.0)$ and $0.1 \mathrm{M}$ sodium chloride at $25^{\circ} \mathrm{C}$. Excitation was at $380 \mathrm{~nm}$. 


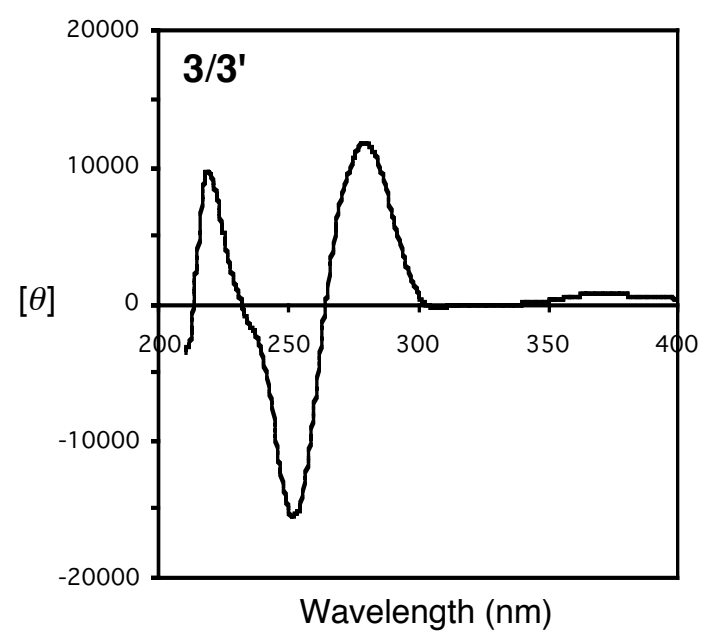

Figure S4. CD spectrum of $2.5 \mu \mathrm{M} \mathrm{3/3}$ in $50 \mathrm{mM}$ sodium phosphate $(\mathrm{pH}=7.0)$ and $0.1 \mathrm{M}$ sodium chloride at $25{ }^{\circ} \mathrm{C}$. The $\mathrm{CD}$ of $230-300 \mathrm{~nm}$ shows that the duplex has B form. The induced CD is observed at $371 \mathrm{~nm}$. This positive CD suggests that the fluorophore is located in the minor groove of the duplex. 


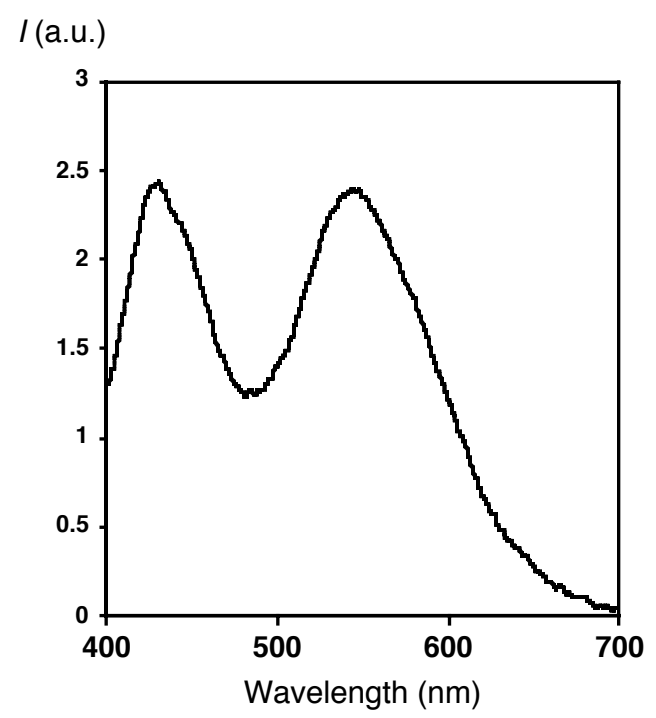

Figure S5. Fluorescence spectrum of $25 \mu \mathrm{M}$ DNA/RNA hybrid, 5'-d(CGCAAT2TAACGC)-3'/5'$\mathrm{r}$ (GCGTTAAATTGCG)-3', in $50 \mathrm{mM}$ sodium phosphate $(\mathrm{pH}=7.0)$ and $0.1 \mathrm{M}$ sodium chloride at $5{ }^{\circ} \mathrm{C}$. Excitation was at $380 \mathrm{~nm}$. 
Table S1. Absorption maxima $\left(\lambda_{\max }\right)$, molar absorption coefficients $\left(\varepsilon_{\max }\right)$, and quantum yields $\left(\Phi_{\mathrm{F}}\right)$ of $2, \mathbf{3}$, and $\mathbf{3} / \mathbf{3}^{\prime}$.

\begin{tabular}{cccc}
\hline & $\lambda_{\max }$ & $\varepsilon_{\max }$ & $\Phi_{\mathrm{F}}$ \\
\hline $\mathbf{2}$ & $375 \mathrm{~nm}$ & 34,100 & $1.02 \times 10^{-2}$ \\
$\mathbf{3}$ & $372 \mathrm{~nm}$ & 26,000 & $7.67 \times 10^{-3}$ \\
$\mathbf{3 / 3}$ & $371 \mathrm{~nm}$ & 21,100 & $7.61 \times 10^{-3}$ \\
\hline
\end{tabular}

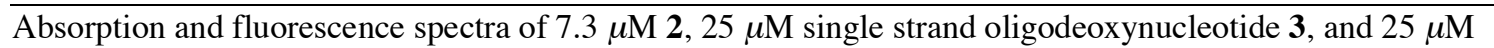
duplex $\mathbf{3} / \mathbf{3}^{\prime}$ were measured in $50 \mathrm{mM}$ sodium phosphate $(\mathrm{pH}=7.0)$ and $0.1 \mathrm{M}$ sodium chloride at $25^{\circ} \mathrm{C}$. 